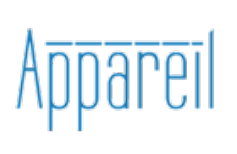

Appareil

15 | 2015

L'art dans le tout numérique

\title{
L'appareil numérique et la perspective ou le retour des espaces projectifs en art
}

Michel Porchet

\section{(2) OpenEdition}

1 Journals

Édition électronique

URL : http://journals.openedition.org/appareil/303

ISSN : 2101-0714

Éditeur

MSH Paris Nord

\section{Référence électronique}

Michel Porchet, «L'appareil numérique et la perspective ou le retour des espaces projectifs en art », Appareil [En ligne], 15 | 2015, mis en ligne le 17 mars 2008, consulté le 03 août 2020. URL : http:// journals.openedition.org/appareil/303 ; DOI : https://doi.org/10.4000/appareil.303

\section{Ce document a été généré automatiquement le 3 août 2020}

\section{(c) (i) (9)}

Appareil est mis à disposition selon les termes de la Licence Creative Commons Attribution - Pas d'Utilisation Commerciale - Pas de Modification 4.0 International. 


\title{
L'appareil numérique et la perspective ou le retour des espaces projectifs en art
}

\author{
Michel Porchet
}

\section{De la technique}

\section{Les systèmes techniques sont finis}

1 Tout outil technique est destiné à agir sur une chose dans un but défini. La chose sur laquelle agit l'outil doit être suffisamment tangible pour inscrire en elle-même la conséquence de l'action. Les systèmes techniques sont strictement finis : leur étendue, leurs résolutions spatiale et temporelle sont finies, leur rapidité limitée, ils ne travaillent ni sans énergie ni à énergie infinie. Les objets de la technique ne sont pas une instanciation médiocre d'un idéal, l'imperfection est consubstantielle à l'action qui les crée. Le tour, par exemple, ne réalise pas le concept de rotation, mais étend l'action plastique du potier. Les systèmes techniques ne tendent pas vers un idéal non fini, ils ne sont valides que sur une échelle étroitement bornée. Agissant dans un monde fini, avec des ressources limitées, la technique écarte infinis et infiniment petits. Les procédures techniques ne peuvent être simplement finies, elles doivent être effectives, celles qui conduisent à des explosions combinatoires sont rejetées. Ces procédures ne laissent que peu de place aux spéculations et doivent autant que possible écarter les débats. La langue des spécifications, des cahiers des charges, des notices, etc., ne doit pas prêter à l'interprétation. Les langues techniques sont donc pauvres par obligation : dominées par le lexique pour les artisans, partiellement formalisées et empruntées à la science pour les ingénieurs. Pauvreté du langage et absence radicale de transcendance sont les sources du faible intérêt de la philosophie pour la technique.

2 La technique ne se préoccupe pas de vérité : la vraisemblance, la pertinence, l'efficacité opérationnelle lui suffisent. L'intuition fondant un geste technique n'est pas 
nécessairement compétente. Le mythe, qui n'exige aucune vérité théorique, se prête à la formulation et la transmission des règles techniques à condition que les assertions fondent le mythe et non l'inverse. Les savoir-faire sont des procédures techniques que l'on ne sait pas expliciter. La bicyclette est un système technique d'apparence simple et familière. Sa conduite n'est pas innée mais une fois l'apprentissage accompli «savoir faire du vélo ne s'oublie pas » comme l'affirme le bon sens populaire. En raison de l'effet gyroscopique, la bicyclette en mouvement est un système mécanique non linéaire dont le comportement, qualifié de "vicieux " par les automaticiens, n'est pas conforme à une intuition simple. Pour tourner vers la droite, il est indispensable d'amorcer un mouvement vers la gauche. Il est donc impossible de s'éloigner d'un trottoir ou d'un rail de tramway si on en est très proche au départ. Adolescent, cela m'a valu un grand nombre de chutes. Conditionné par la mécanique du point matériel, j'attribuais ce comportement non à la bicyclette mais à un défaut de la volonté de celui qui la chevauchait. J'ai multiplié les tentatives, donc les chutes. Peu de cyclistes sont conscients de ce phénomène, et une infime minorité est capable de l'expliquer. L'utilisateur compétent n'adapte pas sa conduite par un mécanisme de "feed back", mais intuitivement. Les automaticiens désignent ce genre de commandes par le terme «a priori ».

\section{La technique industrielle}

3 La technique industrielle agit dans le cadre de procédures explicites, qui sont des programmes. L'industrie ne se caractérise ni par la masse (l'industrie lourde), ni par le nombre (la grande série), ni même par la matérialité des choses sur lesquelles elle agit. La tendance à programmer activités et développements fonde le rationalisme industriel et sépare l'industrie de l'artisanat. Le caractère fondamental de cette tendance n'était pas pensable au $19^{\mathrm{e}}$ siècle et le marxisme l'a perçue de façon non critique (Lénine admirait le système de Taylor).

4 Si tout geste technique artisanal poursuit un but et est en ce sens utile, l'action ellemême n'est pas finalisée et son produit n'est pas nécessairement dirigé vers l'utile. Par contre, l'action technique industrielle, très largement finalisée, est toujours la mise en œuvre d'un programme plus ou moins local (par exemple les gammes opératoires élaborées par les "méthodes »). Les programmes, et non le programme (la pluralité est ici décisive), ne forment pas un plan mais subissent la concurrence d'autres programmes ainsi que des contraintes sociales (le rapport de force entre le capital et le travail) et politiques (lois, normes, règlements, taxes, subventions). Régulés par le marché, ils sont révisés et réadaptés en permanence. Un programme n'est pas impératif, sa mise en œuvre n'est pas son exécution, à la manière d'un ordinateur, elle exige un consensus. Les tensions, socialement déterminées, entre le programme et sa mise en œuvre doivent être limitées pour éviter la mise en danger du but.

5 L'industrie déteste les savoir-faire dont la captation, donc l'inclusion dans les programmes, est difficile. Les hommes qui interviennent dans le processus industriel et se soumettent à lui sont des agents anonymes interchangeables, des prolétaires et non des singularités. Mêmes anonymes et interchangeables, ces agents ont, ne serait-ce qu'en raison de leurs savoir-faire, une certaine autonomie (en partie conquise, en partie octroyée). Ils ont des décisions à prendre. Le professionnel ${ }^{1}$ qui met en œuvre des techniques est l'interlocuteur privilégié de l'utile. Porteur du «marché » (il est salarié 
ou facture sa prestation) et détenteur de savoir-faire qu'il valorise, il respecte la norme. La mise en œuvre professionnelle de la technique intègre les limites de celle-ci plus qu'elle ne les questionne. Convaincus qu'ils font mieux, ce qui est souvent vrai, que les techniques émergentes, les professionnels deviennent, dans leur grande majorité, gardiens du bon usage et de la reproduction de cet usage. Ils se soumettent aux programmes.

6 Selon Habermas ${ }^{2}$, un comportement incompétent, enfreignant des règles techniques éprouvées, est normalement condamné à la faillite par l'échec. Un mécanisme, comparable à la sélection naturelle, entraîne la consolidation et l'évolution lente des techniques. Habermas surestime le côté impératif des règles ou des stratégies. L'art du technicien réside dans sa capacité à saisir le moment où il faut transgresser les règles. Les vrais changements sont le fruit de la rencontre de circonstances exceptionnelles et d'individus singuliers violant les règles du bon usage technique et s'émancipant, partiellement, de leur aliénation au programme. L'histoire de l'informatique fournit nombre d'exemples: les créateurs d'Unix étaient payés par ATT pour développer un système de traitement de texte; des jeunes chercheurs universitaires, s'écartant du cœur de leurs recherches au désespoir de leurs directeurs de thèses, ont élaboré les outils de base de l'Internet ; le monde du jeu vidéo a fait appel, dans sa phase artisanale, à des adolescents passionnés, qui faisaient des choses jugées impossibles par les experts.

7 L'automatisation ne peut s'appuyer sur des mythes, elle exige des modèles fondés théoriquement, au moins localement. Elle met fin au consensus, le programme devient impératif, son exécution ne saurait être négociée. L'évolution récente de l'industrie automobile éclaire ce genre de changement. Le rejet, par une classe ouvrière encore forte, du fordisme (caractérisé, entre autres, par la chaîne dont le défilement détermine l'exécution du programme) a conduit, dans les années 1980-1990 à un éclatement en unités opérationnelles plus petites, à la robotisation d'une partie des activités et à une organisation plus consensuelle des tâches. Ce type d'organisation, que l'on peut désigner par le terme "toyotisme» (en référence à la firme Toyota qui en fut l'initiatrice), s'est retourné, en raison du renforcement d'un chômage structurel et d'une série de défaites majeures des travailleurs, pour devenir une des méthodes les plus dures d'exploitation.

\section{Modélisation et numérisation : mots clés de la technique contemporaine}

8 Les technologies qui ont émergé dans les dernières décennies du $\mathrm{xx}^{\mathrm{e}}$ siècle ont leurs racines dans le développement massif de la technoscience : soit d'une « science » qui ne se préoccupe plus de son rapport à une "vérité ». Cette évolution n'est pas une conséquence inéluctable du développement des forces productives et de rapports de production qui en découlent. Singulière, elle est directement liée à l'enchaînement de la deuxième guerre mondiale et de la guerre froide. Précéder l'Allemagne nazie, dans la mise au point de l'arme nucléaire, justifiait la mise en œuvre de moyens énormes. La peur atomique, le risque d'une guerre totale entre les deux blocs a ensuite justifié la poursuite de l'effort entrepris. Ce modèle n'est plus actuel. La recherche industrielle, finalisée a pris le dessus sur la recherche dite « fondamentale ». La victoire de Microsoft sur les géants du complexe militaro-industriel en est le signe le plus évident. 
9 L'Internet est présenté comme une, sinon La forme la plus avancée de la modernité. Pourtant, en tant qu'utopie, la toile appartient déjà au passé. Confronté au réel, qui résiste, il est tentant de fuir dans le virtuel. Cette utopie réunissait des individus décidés à se défaire des limites imposées par la nature, le corps et la société ${ }^{3}$ et des marchands décidés d'émanciper la marchandise des pesanteurs et limites de la matière. Le réseau a permis de remettre en cause les structures pyramidales et d'envisager d'autres approches des relations de pouvoir. Vaincues, les utopies se retournent contre leurs auteurs. L'effondrement de la "net economy», les irruptions de la propriété intellectuelle, du droit des marques, des mécanismes de censures montrent que la fin de la récréation a été sonnée. Plus personne n'envisage que la "nouvelle économie » domine et asservisse l'économie traditionnelle. Le pouvoir direct des détenteurs du capital et des contenus culturels s'exerce pleinement sur les entrepreneurs du virtuel. Le domaine des réseaux est de plus en plus pris en charge par des ingénieurs traditionnels, issus des écoles de télécommunications. Fiables et prévisibles comme des centraux téléphoniques, ils font plus sérieux que les barbus en sandale qui ont créé l'Internet. La prise de contrôle de l'Internet, par les industries culturelles, en fait un marché où des clients isolés achètent des contenus multimédias à des prestataires professionnels.

10 Le paradigme cybernétique a dominé les automatismes de la deuxième moitié du $20^{\mathrm{e}}$ siècle. La cybernétique décompose: acquisition > transport > interprétation/ traitement > action. L'acquisition d'une information, ou l'action, se fait par un capteur, respectivement un actionneur physique, qui a besoin d'une certaine énergie pour réagir et qui exige inévitablement un seuil d'intensité du signal et un délai. Le transport du signal est source de bruit, le traitement exige un modèle. La cybernétique est une science des modèles qui traite du transfert d'informations entre machines. Elle utilise l'analogie comme méthode de recherche et principe de raisonnement et analyse quantitativement les rapports entre les variations des entrées et des sorties d'un système. Sans les équations différentielles qui expriment, en fonction du temps, les influences mutuelles elle n'est qu'une coquille vide.

11 Le « temps réel » n'est pas celui de la réalité. Cette notion, appartenant au paradigme cybernétique, n'acquiert un sens qu'en relation à une famille d'actions sur un système défini. Il faut donc bannir « le temps réel » pour ne retenir que l'expression « en temps réel » appliquée à une action définie. La définition la plus restrictive de «en temps réel ", indépendante de toute notion absolue de vitesse, se fonde sur la capacité d'agir sur le comportement d'un système cybernétique (ou en d'autres termes bouclé ou possédant une contre-réaction). De façon plus laxiste, c'est un quanta de temps, un instant de durée finie d'observation d'un phénomène et d'action sur ce phénomène. Un système informatique est dit «en temps réel » si le délai de sa réaction à une requête d'interruption est déterministe. Par un abus de langage, on désigne du même terme des systèmes dont la réponse à une question complexe est suffisamment rapide pour que les délais soient acceptés par un utilisateur.

12 Si les programmes visent à agir sur des phénomènes, leurs développements ne peuvent être fondés sur les phénomènes eux-mêmes: les modèles sont d'indispensables médiateurs. Un modèle est toujours in fine, une abstraction, un objet mathématique, une projection « orientée problème » de la réalité. La construction d'un modèle revient à considérer comme seuls pertinents certains phénomènes. L'espace de modélisation est situé dans un monde dont les limites doivent être clairement définies. Pour être 
performant, le modèle doit être composé d'objets jouissant de propriétés utilisables. Différentes linéarisations (globales ou locales) permettent de le simplifier le plus possible. Il doit avoir une relation intuitive avec l'objet qu'il représente. Enfin, il est prudent d'envisager des conséquences de l'approximation intrinsèque à toute modélisation sur le statut de vérité des propositions touchant objet réel et modèle. La théorie des graphes, qui décrit des espaces réticulés d'états et de transformations, est un outil privilégié de modélisation. Les espaces qu'elle décrit sont très différents de l'espace euclidien. Elle a son origine dans le problème des ponts de Königsberg étudié par Leonard Euler au XVIII ${ }^{e}$ siècle. Une représentation topographique simplifiée permet de poser le problème.

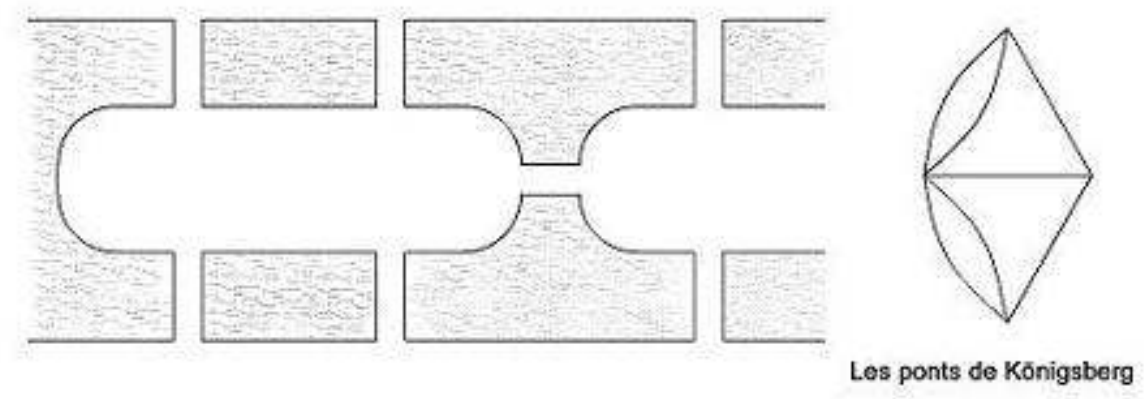

13 Existe-t-il un chemin permettant de parcourir les sept ponts sans passer deux fois sur le même? Pour résoudre le problème, Euler, s'écartant de la géométrie euclidienne, modélise le schéma par un graphe dans lequel, les terres sont réduites à des points (les sommets), les ponts à des arcs. Le degré d'un sommet est le nombre d'arêtes qui le touchent (ici, 3, 3, 3, 5). Chaque passage par un sommet détruit deux arêtes. En dehors des points de départ et d'arrivée, tous les sommets doivent donc être de degré pair pour que le problème ait une solution. Ce n'est à l'évidence pas le cas pour les ponts de Königsberg. Une méthode essai/erreur aurait exigé plus de 500 tentatives pour établir l'absence de solution. Le graphe est une abstraction, un modèle graphique formalisé de la ville de Königsberg. C'est l'apparition d'une appréhension réticulée de l'espace, fort éloignée d'une intuition fut-elle a priori. L'espace dans lequel vit l'homme contemporain est en grande partie réticulé. Il suffit de penser aux transports en commun, aux routes et autoroutes, aux couloirs aériens, mais aussi au téléphone et aux médias, à la distribution de l'énergie et de l'eau. La théorie des graphes ne s'est développée que dans les années 1950, en relation avec la logistique (le célèbre problème $\mathrm{du}$ voyageur de commerce), la recherche opérationnelle et les besoins de la programmation.

\section{L'industrie : de la transformation à la synthèse}

La technique industrielle a évolué d'une technique de transformation à une technique de synthèse. Bachelard a parlé remarquablement de la synthèse en chimie ${ }^{4}$. Même si certains disciples de Derrida haussent les épaules et affirment qu'il a été "déconstruit", Bachelard est un des seuls philosophes français ${ }^{5}$ à avoir pensé la technique industrielle avec quelque pertinence. Aujourd'hui, on veut parvenir à une synthèse programmée exécutée par un système technique donc fini. Cette synthèse ne 
saurait être l'inversion de l'analyse car une telle inversion fait toujours apparaittre des combinatoires. La synthèse, par un système technique, impose soit une méthode dite d'identification ${ }^{6}$, soit une démarche "constructive ». Les démarches "constructives " conduisent à des problèmes de l'ordre de ceux posés par le langage, en particulier les explosions combinatoires. C'est entre ces deux pôles qu'oscillent les solutions techniques avec aujourd'hui, par l'intermédiaire des programmes, une domination du deuxième pôle. L'impuissance du traitement mécanisé face à l'explosion combinatoire impose une réduction extrême des modèles qui finissent par ne plus rien nous dire du monde. L'affirmation de Barthes: «Bien que toute création soit nécessairement une combinatoire, la société, en vertu du vieux mythe romantique de l'inspiration, ne supporte pas qu'on le lui dise $»^{7}$ laïcise la création mais ne dit rien des démarches effectives qui y conduisent. L'existence de combinatoires impose une vitesse de calcul et une mémoire non pas très grandes, mais aussi proches que possible de l'infini. Face à l'explosion de la combinatoire, le tâcheron fait appel au stéréotype, au lieu commun, à l'habitude, au cadre que lui ont enseigné ses maitres. Il débouche sur une solution banale. Le concepteur talentueux trouve quelque chose, de l'ordre de la distance juste, pour entrevoir un chemin nouveau et conserve une probabilité non nulle de déboucher sur une solution qui ne soit pas banale. Comment trouve-t-il cette «distance»? On n'en sait rien. On retrouve, sous une forme à peine modifiée, la question initiale ${ }^{8}$.

À l'ère des ordinateurs et des réseaux, la technique tente d'opposer une puissance combinatoire aux problèmes soulevés par les combinatoires. Des chercheurs recherchent les solutions aux questions sur l'origine des comportements du vivant dans la tactilité, la plasticité du comportement de "bestioles », dotées de certaines capacités d'apprentissages et confectionnées avec des éléments électromécaniques de vieux ordinateurs et une logique limitée à quelques centaines de transistors. Un grand nombre d'agents autonomes très simples, poursuivant un but individuel lui aussi très simple (survivre, se nourrir, se reproduire, etc.), interagissent. Au-delà d'un certain niveau de complexité, des comportements, pertinents dans certaines situations, apparaissent que l'on n'aurait pu déduire de la connaissance des éléments individuels. Dans un système suffisamment complexe, un mécanisme de sélection, légèrement supérieur du point de vue d'une évolution positive au choix au hasard, garantit une organisation croissante. Cela traduit l'idée que l'information est une entropie négative. Cette conception, qui fait dériver l'évolution de programmes capables de s'adapter et se complexifier, plus radicale encore que la Gestalt, entraîne une pensée différente de la relation entre la partie et le tout. Elle a modifié notre vision des colonies d'insectes sociaux. Les techniciens évitent d'affirmer que leurs « animalcules» vont parvenir au langage puis à la conscience. Cette prudence est motivée par les affirmations, aujourd'hui ridicules, tenues il y a cinquante ans par Simon, inventeur avec Newell et Shaw de l'Intelligence artificielle : « Nous avons [...] ainsi résolu le vénérable problème esprit/corps, expliquant comment un système composé de matière peut avoir les propriétés de la pensée. $»^{9} \mathrm{Il}$ n'en reste pas moins que la technoscience tend toujours, par ses fondements même, à réduire la pensée à une question purement quantitative, à un simple saut de complexité. 


\section{De l'espace et du temps}

\section{La perception}

16 La perception humaine ne peut être réduite aux cinq sens. Au-delà de ce qu'on appelle les sens, l'homme perçoit ses mouvements, ses postures, les champs thermiques, le magnétisme, et certains rayonnements. Les deux derniers groupes de phénomènes n'ont pas une relation claire avec le système nerveux central, il est donc concevable d'affirmer que nous les subissons plus que nous les percevons. Par contre, la maîtrise de nos mouvements a une relation directe à notre perception du monde. La perception dépend aussi de nos activités, de nos moyens d'expression et de communication. «Une conscience qui n'aurait pas la capacité d'imaginer ne percevrait rien. $»^{10}$

Le présent d'un être, doté d'un système nerveux évolué et d'une capacité mnémonique, se situe dans une tranche de temps qui n'est pas l'instant, le présent au sens strict, mais une partie du passé totalement rémanente et un morceau d'avenir totalement prévisible. Sans ce fragment de temps, qui n'est pas inné mais intègre notre expérience sensible et se règle sur nos activités, il n'y a ni perception ni action. Avant sa naissance, l'homme perçoit les battements du cœur de sa mère. Comme tous les mammifères, il est donc confronté à un temps cyclique durant une partie de la gestation. Ce rythme, perdu à la naissance, ne se retrouve que dans des moments privilégiés, lorsque le nourrisson est couché sur la poitrine de sa mère. Il joue un rôle dans la construction de notre inconscient. La seconde est le temps de ce rythme, très proche de celui des actions interactives en "temps réel». Ce n'est que plus tard que l'enfant "constate» l'existence de phénomènes irréversibles, qu'il peine à accepter. Même l'adulte qui, par sottise ou maladresse, casse un objet auquel il tient, veut, pour une fraction de seconde, revenir en arrière et annuler le geste néfaste.

La perception de l'espace proche est visuelle et tactile. L'œil comporte deux ensembles de capteurs interconnectés (l'un pour la perception fine des formes, l'autre pour celle des couleurs). La découverte de l'espace proche par le toucher suppose des conditions de taille et de complexité géométrique. Le toucher est le seul de nos sens qui soit réciproque, « toucher est le même geste que d'être touché " ${ }^{11}$. La perception de l'espace lointain, de l'étendue, est visuelle, sonore et ergotique. Sans l'effort, sans une certaine dépense d'énergie musculaire, la mémorisation de l'exploration d'un univers reste médiocre, l'information perd sa consistance pour le système nerveux. C'est la dimension ergotique. L'intervention du corps, de la réalité du temps et de l'effort physique et mental, rend totale la perception de l'environnement.

19 La perception civilisée s'écarte de la perception sauvage. L'ouïe est le sens qui perçoit l'espace qui nous entoure comme une totalité et le surveille de façon permanente. Besoin absolu pour le chasseur/proie primitif, ce sens sonore de l'espace est beaucoup moins important pour l'homme civilisé dont l'ouïe perçoit surtout la parole. Le sens spatial du son finit de se perdre dans une écriture purement séquentielle. C'est aussi par une écriture séquentielle que la musique est devenue paradigme des phénomènes perceptibles temporels. Les théories physiques et physiologiques du son s'appuyant sur les ondes stationnaires (Helmholtz) ne remettaient pas en cause cette conception. Des conventions scénographiques (disposition de l'orchestre, lieux reconnus propres à tel genre de musique) garantissaient la permanence de l'expérience spatiale pour 
l'auditeur. La synthèse musicale a imposé une critique approfondie des théories analytiques et la construction de nouvelles théories de la perception du son musical ${ }^{12}$.

\section{L'abstraction}

La conscience accède à l'instant et au point par la médiation d'abstractions. Si ces abstractions sont fertiles pour la mathématisation du monde, elles ne sont pas indispensables à sa connaissance ${ }^{13}$. La géométrie a évolué d'une science des figures à une science de l'espace, puis d'une science de l'espace physique à la science abstraite de l'espace. Dans le programme d'Erlangen (1872), Félix Klein ${ }^{14}$ a exposé une classification des géométries fondée sur les invariants associés à un groupe de transformations. D'abord instruments, les transformations deviennent l'essence d'une géométrie science abstraite de l'espace. La notion moderne d'espace géométrique s'articule sur le continuum mathématique et la notion de coupure due à Dedekind. Pour les physiciens contemporains, tentant d'unifier relativité et théorie quantique, espace et temps sont des phénomènes, manifestations de processus plus profonds, qui se mettent en place dans l'évolution même de l'univers. Il est toujours possible d'affirmer comme Cassirer, que $\mathrm{Kant}^{15}$, bien qu'il invoque la géométrie euclidienne et l'arithmétique pour fonder l'intuition a priori de l'espace et du temps, n'est pas réfuté par la physique contemporaine, les argumentations étant incommensurables. Il n'en reste pas moins qu'espace et temps, loin d'être donnés a priori, sont devenus objets de science.

\section{Le cerveau}

21 L'homme vit en société et la pensée s'appuie sur le logos, moyen de communication. L'homme isolé de la nature, puis de son espèce est un paradigme orgueilleux de la science occidentale, consacré par le cogito cartésien. Le type de décomposition qui fonde le modèle cybernétique ne s'applique que médiocrement au vivant. Toute réflexion doit prendre en compte la manière dont les structures psychiques, le sexe, la position hiérarchique mais aussi les contraintes du corps déterminent la perception. Le même cerveau gère fantasmes, spéculations rationnelles, et perceptions. Il intègre nos expériences réelles ou fictives, les premières se distinguant surtout par leur caractère total. L'imagination ne relève pas du raisonnement mais d'une capacité mentale de projection dans des situations réelles ou hypothétiques. La même capacité mentale est à l'œuvre dans la lecture des fictions et dans la compréhension d'autrui. Cela donne au cerveau une capacité d'anticipation utilisée par tous nos comportements compétents. Le cerveau est le produit d'une longue évolution, qui ne fait jamais table rase, mais construit au-dessus de l'existant. Le mécanisme naturel de sélection et de spécialisation ne met pas en œuvre la combinaison d'un nombre limité d'atomes mais crée des éléments très divers. La très complexe organisation spatiale du cerveau reproduit, dans une certaine mesure, celle du corps. Il y a, par exemple, une aire corticale qui reflète la structure de la main. Ce genre d'organisation spatiale se retrouve dès la partie du génome qui concerne la mise en place de l'ensemble bras/main. Les composants de notre système nerveux sont lents. Les phénomènes élémentaires au niveau des synapses ont des constantes de temps de l'ordre de $10 \mathrm{~ms}$. Cette lenteur n'empêche pas le système nerveux d'être très rapide. Le très fort parallélisme de son organisation ne suffit pas à expliquer les performances du cerveau. «L'évolution a fait du cerveau une 
machine à prédire et non pas un comptable des situations ${ }^{16}$. Il y a anticipation, choix à l'intérieur du sous-ensemble pertinent a priori d'un ensemble d'actions préparées.

La mémoire n'est pas réversible, l'oubli n'est pas l'ignorance. Le cerveau intègre l'ensemble de nos perceptions et spéculations de façon irréversible. Il est archive d'un vécu socialisé et inscrit dans une histoire. Les systèmes «mécaniques » de calcul sont fondés sur des composants banalisés travaillant à des fréquences très élevées. Totalement réversibles, ils ont une mémoire mais pas d'histoire. Leur fonctionnement en parallèle exige une horloge et l'échange d'un grand nombre d'informations de synchronisation (d'ailleurs indispensables à la réversibilité). Cette réversibilité rend suspectes les prétendues archives que ces systèmes peuvent enregistrer. Elle permet aussi un apprentissage au travers de méthodes essais/erreurs qui ont toujours pour conséquences de soumettre l'utilisateur à la machine.

\section{Le cinéma}

\section{Un spectacle de masse}

Dans le spectacle vivant, l'écriture est préalable, l'interprétation, la représentation et l'observation sont simultanées. La reproduction mécanique va à la fois permettre et imposer de séparer les temps de l'interprétation et de la représentation. Le processus est :

Écriture > interprétation, action, captation > montage > représentation.

Interprétation, action et captation sont simultanées. W. Benjamin souligne que le jeu artistique de l'acteur de cinéma se différencie de celui du comédien de théâtre car il a lieu non face à un public mais face à des professionnels :

[...] En élargissant le champ soumis aux tests, le rôle des appareils par rapport à l'interprète est analogue à celui que jouent, pour l'individu, les conditions économiques qui ont augmenté de façon extraordinaire les domaines ou il peut être testé. Ainsi voit-on les épreuves d'aptitude professionnelle prendre de plus en plus d'importance. Elles consistent en un certain nombre de découpages opérés dans les performances de l'individu. Prise de vues cinématographique, épreuve d'aptitude professionnelle, l'une et l'autre se déroulent devant un comité d'experts. Le chef opérateur, au studio, occupe exactement la même place que le directeur des tests, lors de l'examen d'aptitude professionnelle. ${ }^{17}$

L'industrie n'est pas inéluctablement une catégorie marchande. Avec Griffith et Eisenstein on est loin de l'artisanat de Méliès. À la poursuite de buts politiques et idéologiques, l'industriel Eisenstein est plus éloigné de considérations commerciales que l'artisan Méliès. Pour lui, le langage cinématographique a la possibilité, à partir de l'émotion esthétique, de développer un discours de type théorique sur les événements historiques et le phénomène révolutionnaire lui-même. Plutôt que d'essayer d'aligner le cinéma sur une discipline déterminée, c'est plutôt au cinéma qu'il voulait confier la tâche de fournir en concepts les discours sur les arts. Le cinéma était un art de masse, les salles obscures sont des lieux où les gens vivent la même chose à la fois ensemble et individuellement. Au début du $\mathrm{Xx}^{\mathrm{e}}$ siècle, masse ne signifie pas clientèle solvable mais partage d'une expérience commune. L'irruption des masses a précédé politiquement et socialement la massification des biens et de la clientèle solvable. Les masses qu'invoque Benjamin - non réductibles au nombre, au peuple ou à la population - étaient une force politique en mouvement: "Celui-ci [l'actuel épuisement de l'aura] s'appuie sur la 
conjonction de deux circonstances qui sont liées au plus haut point à l'épanouissement croissant et a l'intensité grandissante des mouvements de masse. ${ }^{18}$.

L'illusion, la confusion entre l'image et un référent, dépend des attentes du spectateur et ne se produit que s'il existe une interprétation plausible de la scène cinématographique. Le spectateur ne croit pas voir le réel, mais quelque chose qui « qui pourrait avoir été» selon la formule de Godard. Industrialisée, l'image cinématographique s'universalise et crée pour tout spectateur un mode unique d'illusion qui semble modifier, perturber, pervertir le rapport au réel. C'est un simulacre. Le spectateur de film, assis dans une salle obscure, n'est en principe ni dérangé ni agressé. Il est complètement enveloppé par des indices de réalité et rendu apte à répondre psychologiquement à ce qu'il voit et imagine.

Godard l'a martelé une fois pour toutes : au cinéma on lève les yeux, devant la télé, la vidéo on les baisse. Plus le rôle de l'obturateur. Sur deux heures passées dans une salle de cinéma, on a passé une heure dans la nuit. C'est cette part nocturne qui nous accompagne, qui "fixe" notre souvenir du film d'une façon différente du même film vu à la télé ou sur un moniteur. ${ }^{19}$

Les conditions dans lesquelles le cinéma se donne à voir ont profondément changé. Des salles de plus en plus petites, des fauteuils conçus de façon à minimiser les interférences entre spectateurs transforment la vision d'un film en expérience singulière, difficile à communiquer, offerte à un grand nombre d'individus. Par le home cinema, le cinéma se montre dans l'univers familier, le téléviseur fait «zapper » entre l'image et le salon. L'expérience partagée est liquidée, et la reproduction de l'œuvre, ainsi que le relève Benjamin « remplace son occurrence unique par une existence en série et en permettant à la reproduction de venir à la rencontre de celui qui regarde en toute circonstance, elle actualise la chose reproduite $»^{20}$. Dans ce dispositif, la synthèse des expériences proposées ne se réalise pas, mais cela n'entraîne pas d'interrogation. Une synthèse n'a, a priori, pas de raison de se produire dans un tel cadre.

\section{Un art du montage}

Dans le dispositif du théâtre à l'italienne, comme dans les baraques de foire, la mise en scène n'a pas une maîtrise complète du point de vue du spectateur. Le volume de la boîte (espace du jeu) s'oppose à celui de la salle (espace du spectateur), la frontalité n'est pas parfaite. Le cinéma et la photographie réalisent le dispositif perspectiviste (observateur, cadre, profondeur de champ, optique de la caméra) et imposent la frontalité. L'auteur maîtrise le point de vue. Il est donc facile de truquer l'espace du champ en agissant sur la taille, l'échelle, la perspective (fausses lignes de fuite), l'orientation (se libérer de la pesanteur en permutant horizontale et verticale). La reproductibilité technique passe par l'inscription, la mémorisation (au moins en un certain sens). L'enregistrement de phénomènes dynamiques permet de truquer la relation entre l'espace et le temps, de rapprocher, voire de ramener en un même espace-temps des traces d'événements ayant eu lieu en des temps et lieux différents. Des temporalités différentes sont combinées en changeant la vitesse de prise de vue.

La persistance rétinienne autorise la décomposition en photogrammes. Le photogramme est un fragment d'un ruban d'étendue finie : le rush. Il est aussi un objet qui peut être montré pour lui-même. Cette décomposition spatialise un « morceau » de temps sur un support unidimensionnel, objet possible de manipulations plasticiennes. L'espace du rush peut être parcouru librement. Il est possible de sauter des fragments, 
d'aller en avant ou en arrière. Le support argentique donne à l'image animée une plasticité qui n'est présente que pour les formes courtes produites artisanalement. Alors que la plasticité se situe dans l'ordre de l'invariance ou de la modification graduelle des topologies, la coupe, action fondamentale du montage, brise continuités et topologies pour construire une structure. Cette tension entre le cinéma et les pratiques de plasticiens a débouché sur l'une des formes du cinéma expérimental. Si le support film se prête aux pratiques plasticiennes, le cinéma est un art du montage : un langage qui emprunte à d'autres sources. C'est dans le cadre du Théâtre itinérant du " Proletkult », qu'Eisenstein a expérimenté son principe du "montage des attractions ", partant des réactions psychophysiologiques du spectateur.

31 Le montage cinématographique se fait sur une copie de travail qui doit être coupée et collée. Ce travail prend du temps, le résultat n'est pas disponible en tout temps. La coupe, action fondamentale du montage, n'est réversible que par collage. Un travail chaotique dégrade très rapidement la copie de travail. Lorsque le montage se fait directement sur l'original (cas des amateurs et des fauchés comme Mekas) le repentir est encore plus limité. L'apprentissage d'un comportement compétent est imposé par la technique. La présence de sons "réels » change un peu la règle du jeu. Le temps de réponse de l'oreille est beaucoup plus court que la durée de la persistance rétinienne. L'ouïe ne supporte pas le montage «cut» du son, il faut aménager des transitions. Si l'image peut être découpée jusqu'au photogramme (qui est un "quantum de temps »), cela devient impossible, avec des moyens analogiques, pour le son qui appartient au continuum. Il n'y a de "grain" de son que suffisamment bref pour rester imperceptible. Le "sonore», dans un premier temps, diminue la capacité de fragmenter le temps, rapprochant ainsi le cinéma du spectacle vivant. Ce n'est donc pas seulement l'irruption du verbe qui ramène le cinéma « parlant » vers le théâtre, mais la perception même du son qui est un obstacle à la liberté du montage.

32 Les limites de la mémoire humaine renforcent le poids de l'écriture à mesure que la durée de l'œuvre augmente. La maîtrise du matériau «images » est impossible sans écriture préalable. L'écriture va jouer un rôle clé dans la façon dont les images seront mémorisées par le réalisateur. Elle guidera les propositions de solutions de montage. Comme toute industrie, le cinéma industriel s'articule autour de programmes. Le film d'Eisenstein La ligne générale est un emblème du grand cinéma industriel. Pour atteindre ses buts, Eisenstein a développé le concept de «montage intellectuel»visant à introduire le spectateur au raisonnement dialectique. «La forme du montage est structurellement une restitution des lois du processus de la pensée, laquelle à son tour, restitue la réalité mouvante en cours de déroulement. $»^{21}$

\section{À propos de la vidéo}

La vidéo, comme le radar, code une exploration de l'espace du champ par un signal électrique structuré et purement temporel. Un grain de temps, très court à l'échelle de nos perceptions, permet de coder la projection de l'espace sur la durée qui sépare deux trames. Contrairement au rush cinématographique, la bande vidéo n'a pas de vie propre, elle doit être décodée. Une bande vidéo c'est du temps, a dit Nam Jum Paik, j'ajouterai du temps sur lequel on a techniquement greffé « une structure d'ordre spatial ». Du point de vue philosophique, il n'y a rien à déduire de cette structure, elle n'est qu'une conséquence d'un processus de codage/décodage qui est l'émergence d'un 
«protolangage » auquel s'applique pleinement la théorie quantitative de l'information initiée par Shannon.

À ses débuts, la télévision démultiplie le spectacle vivant par sa diffusion en direct, un peu comme le réseau téléphonique parisien a servi dans un premier temps à diffuser des concerts. Faute de " mémoire ", de surface d'inscription, elle n'est pas un moyen de reproduction. Pour s'affranchir du direct, la télévision ne disposait que du télécinéma. Avant la généralisation du magnétoscope, la vidéo ne sait pas manipuler le temps. A la prise de vue, la sensibilité trop grande des détecteurs limite l'ouverture, donc la capacité de jouer de la profondeur de champ. Le grain de l'image mais surtout une absence de «matière » handicapent l'image vidéo. Le faible rapport de lumière entre le blanc extrême et le noir le plus noir (le rapport signal/bruit) entraîne de faibles nuances des blancs et des noirs. La radicale diminution du grain de temps (l'augmentation de la fréquence du signal) a entraîné, dans un premier temps, une limitation des possibilités de montage. On ne s'arrête pas plus sur une image vidéo que sur un son, l'acuité de la coupe est diminuée. Le médiocre rapport signal/bruit limite le nombre de générations, donc les effets. Les difficultés de synchronisation rendent médiocres les superpositions. Sous une apparente légèreté se cache une grande prégnance du dispositif. Le magnétophone a apporté très rapidement un rapport nouveau entre le son, la musique et le temps : l'émergence d'un matériau sonore, qu'il a été possible de travailler dans un autre temps que celui de sa diffusion. Cette nouvelle modalité d'inscription a débouché sur des formes radicalement nouvelles, la musique concrète, l'acousmatique et la musique électronique. On ne peut en dire autant de la vidéo. Précédée et conditionnée par la photographie et le cinéma, elle est, à ses débuts et en dehors de quelques œuvres singulières, restée faible dans l'émergence de nouvelles propositions d'images.

La numérisation du signal permet de retrouver certaines possibilités du cinéma. L'existence d'un grain de temps (l'inverse de la fréquence d'échantillonnage) et la discrétisation des amplitudes des signaux donne accès à un quantum informationnel : le pixel. Les pixels sont organisés en trames auxquelles on accède par une adresse : le « time code ». Dans une régie numérique, une « liste de montage » (« edit list») ordonne les différents fragments de l'objet audiovisuel. Les possibilités de trucages de montage et d'étalonnage deviennent très grandes. Le repentir prend la forme d'une modification de la liste de montage. Pour voir le résultat, il faut confectionner une bande, par une procédure automatisée. Par contre, du fait de la numérisation, cela se fait sans dégradation du matériel. Les caméras sont dotées de très nombreuses possibilités de réglage et visent une qualité de l'image en gros comparable à ce qu'offre le $16 \mathrm{~mm}$. Beaucoup de défauts de la vidéo paraissent ainsi dépassés.

\section{La surface d'inscription : du ruban, à l'accès aléatoire}

Pur objet théorique destiné à étudier les structures algorithmiques, la machine de Turing est pensée sans limites. Son dispositif mémoriel est un simple ruban porteur de signes qu'elle peut écrire, lire et effacer. Cette architecture ne pose aucun problème dans un monde de ressources infinies: le temps d'accès à une donnée reste nul quelle que soit la longueur d'un ruban que rien ne limite. Il n'en va plus de même dans un monde fini. Dans le monde réel, on souhaite pouvoir accéder à des données le plus vite possible et de façon aussi indépendante que possible de leur localisation sur le support 
d'enregistrement. La surface d'inscription du cinéma et de la vidéo est un ruban que l'on peut admettre unidimensionnel: il n'existe qu'un chemin d'accès aux données, d'où une forte dépendance du temps d'accès à la position du photogramme ou de la trame. Une structure multidimensionnelle de la mémoire multiplie les chemins d'accès (la RAM, la surface du disque, une mémoire tridimensionnelle, etc.). Il est ainsi possible d'accéder à n'importe quelle trame dans un temps bref et variant peu. L'accès est dit aléatoire. C'est le cas dans le montage virtuel. La «liste de montage» devient une véritable partition actionnant les différents fragments de l'objet audiovisuel. Une modification du montage ne modifie que la partition, il n'y a ni déplacement des objets, ni création de nouveaux objets. Il est possible de voir immédiatement les conséquences d'une modification. Cela reste vrai pour des effets, en ajoutant leur description à la « liste de montage ». Il suffit que la machine soit assez puissante pour les exécuter en «temps réel ».

Ces possibilités sont suffisamment séduisantes pour s'appliquer au cinéma aussi. La copie de travail est remplacée par une copie vidéo numérique sur laquelle se fera le montage. La pellicule étant, elle aussi, "time codée », le négatif pourra être monté à partir de la «liste de montage». Le montage se fait sur une version dégradée de l'image, ce que certains cinéastes, comme Straub et Huillet, jugent intolérable. Il faut dire que, chez Straub-Huillet, la rigueur de l'intention limite considérablement le besoin en prothèses d'un cerveau qui reste la plus puissante machine de montage virtuel. La flexibilité du montage virtuel semble ouvrir la porte à tous les possibles, mais c'est la fin de la plasticité d'un travail de montage qui n'est plus qu'écriture. En réalité, la porte s'ouvre sur un gouffre : toute intention devenant possible et réalisable, l'intention disparaît. Face à la " page blanche ", le recours à l'écriture (à une partition) est indispensable. La métaphore de la "page blanche » est dangereuse. L'écran vide dissimule des procédures programmées susceptibles de déterminer, sans qu'il en soit conscient, les choix de l'utilisateur.

\section{Le passage aux univers virtuels : la « libération » du spectateur}

Le montage virtuel permet une inversion de la façon de penser le déroulement temporel de l'œuvre. La "liste de montage» définit des commandes exécutées séquentiellement. Avec un matériel permettant une exécution en «temps réel » de ces commandes, il devient possible de confier au spectateur le choix de la séquence.

Le virtuel n'est pas le faux, l'illusoire, le trompeur, mais un mode particulier d'existence de la réalité. Germe de devenir qui échappe au temps et à l'espace, il s'oppose à l'actuel. Tant qu'elle n'est qu'un ensemble plus ou moins structuré de données, l'image informatique est virtuelle. L'application, à ces données, d'un certain nombre de méthodes de traitement permet d'afficher ou d'imprimer une image, ainsi devenue actuelle. Les données sont produites par un modèle ou par la saisie d'informations sur un environnement donné à l'aide des capteurs de grandeurs physiques. Une image de synthèse est une simulation, une fenêtre ouverte à la perception d'un monde des modèles dont l'intelligibilité reste virtuelle. Images et sons de synthèses, contrairement aux images argentiques et aux sons des instruments traditionnels, s'appuient inévitablement, par leur caractère même, sur une théorie de la perception. S'il doit prendre en compte cette théorie, l'artiste n'a pas à s'y soumettre. "Voilà pourquoi nous devons déclarer qu'il existe une certaine différence, qu'il y a 
même une grande contradiction, entre le travail créateur du peintre et l'action purement optique. Les preuves optiques ne peuvent servir de loi aux gammes picturales du peintre $»^{22}$.

La modélisation tridimensionnelle libère l'observateur et permet à l'utilisateur de choisir un point de vue. Dans un univers dit virtuel, on simule un modèle d'un monde et le spectateur dans ce monde. L'utilisateur peut agir, dans une certaine mesure, sur son environnement. Cette action peut s'assimiler à la maitrise des mouvements d'un « corps» d'emprunt. Il y a une immersion, mais dans l'espace d'une scénographie. Le spectateur vit l'expérience décevante du simulacre. Dans ce monde de points situés en dehors de lui, il ne perçoit pas sa propre étendue. Il est privé de corps et des organes qui l'ouvrent au monde et lui permettent d'être à sa propre expérience. Le terme "scénographie " n'est pas une métaphore. Dans les images de synthèse, la lumière ne dévoile pas mais cache la nature du modèle. Elle joue les mêmes rôles qu'au théâtre: attirer l'attention sur ce que l'on veut montrer, cacher ce qui ne doit pas être vu, magnifier des décors construits à moindres coûts. L'espace, fondé sur un modèle, pose une question, non résolue, de distance : il programme la pratique de son exploration. Images binoculaires et sons y sont simulés, le toucher fait défaut ou est réduit à sa caricature haptique, la dimension ergotique est absente. Même les systèmes les plus évolués de "réalité virtuelle » restent loin de l'intelligence du geste. Une simulation à la fois insuffisante et trop prégnante ne permet pas à l'imaginaire de se déployer. Au cinéma, l'auteur libre de jouer de l'espace et du temps, proposait au spectateur, contraint à la frontalité et au temps de la projection, plus qu'un récit, une expérience "spatio-temporelle ». L'immersion, nommée à tort interactivité, inverse ce rapport de liberté. Dans un monde de modèles, espace et temps deviennent des conventions. La seule limite est la crédibilité, en termes de règles du jeu, de l'univers proposé. Trop complexe, cet univers ne peut être exploré, pour se mouvoir dans le monde simulé, l'utilisateur a besoin d'une intuition a priori. Cette intuition n'est pas celle invoquée par Kant, mais le recours à des stéréotypes culturels largement diffusés (comme les livres de Stephen King), à la vulgate scientifique ou à des règles élémentaires, du genre « tire sur tout ce qui bouge ». L'espace proposé se fonde sur des appareils éprouvés : il est une métaphore de la bibliothèque, du musée ou de la guerre. La machine géométrique incluse dans le dispositif est perspectiviste. Le média dominant, le cinéma, impose ses codes.

\section{L'installation : une autre règle du jeu}

41 Le dispositif du cinéma a tôt été interrogé par des projections multiples (Abel Gance) visant à faire du spectateur un protagoniste actif. La projection a été explorée par Moholy-Nagy avec le Polykino ${ }^{23}$ mais aussi avec Licht Requisit (1922-1930). Installé, le cinéma offre une autre vision remettant en cause non seulement la frontalité mais aussi la distance. La perception de l'espace varie avec la distance à l'objet: de près la longueur domine; de loin ce sont les angles. Ce que Matisse nommait l'« amplification de l'image » n'est pas une homothétie, un agrandissement. L'image proche et de grand format perturbe une représentation qui doit, elle aussi, être perturbée.

L'installation, alternative à l'immersion, n'est pas une scénographie, le spectateur s'y engage avec son corps. Il y a une totalité de l'expérience, une authenticité de l'interactivité, qui explicitent la tension entre le réel et le simulacre et démasquent ce 
dernier. L'action de l'image reste extérieure au spectateur. Il ne peut se projeter dans un écran inscrit dans l'espace qu'il explore. L'installation avec images et sons affirme son espace propre au détriment de celui du musée. Alors que l'installation " classique " organise la rencontre entre un lieu et un concept, l'installation avec projection d'images et de sons affirme son propre espace et cohabite mal avec d'autres installations du même type. Il lui faut un espace pour le système de projection. La pénombre est nécessaire à la perception de l'image, un relatif silence à celle du son.

Mécanique, la machinerie du cinéma se dévoile en se montrant. Comme le souligne Dominique Païni ${ }^{24}$ citant Foucault ${ }^{25}$ il faut, à la manière de Raymond Roussel, montrer le procédé. Support d'une opération abstraite de codage/décodage, la machinerie vidéo ne démasque rien en se montrant. Pour la vidéo, l'installation est le chemin pour s'émanciper de la télévision et du cinéma.

\section{La relation de l'art à la science respectivement à la technique}

\section{La modernité et la perspective}

Les relations entre l'art du Quattrocento et la révolution scientifique galiléenne sont souvent présentées comme consubstantielles à la modernité. Si Dürer et Copernic sont contemporains, près de deux siècles séparent Brunelleschi et Alberti d'une part de Galilée et Kepler d'autre part. Les peintres n'ont pas attendu les mathématiciens pour développer la perspective. Della Pictura d'Alberti date de 1436, il faudra attendre près de deux siècles pour que Desargues définisse un cadre mathématique correspondant.

Les relations entre l'art et la science sont plus subtiles qu'une ascendance. Il y a un cousinage des problématiques. C'est dans le domaine des idées, de la connaissance en général qu'il faut chercher des liens. Goodman ${ }^{26}$ souligne que l'art a, comme la science, une fonction cognitive. Pour qu'existe une véritable communauté de projet cognitif, il faut qu'existe une forme d'humanisme. L'exposé littéraire de la recherche scientifique, visant l'honnête homme du temps, n'est plus une tâche qui va de pair avec la réflexion elle-même. Il a disparu après la première guerre mondiale, remplacé par la médiatisation de la science. En tant qu'outils de connaissance l'art, la technique, la science et la philosophie répondent aux questions du temps mais à des niveaux et avec des délais très différents. Artistes et scientifiques ne parlent pas de la même chose. La science raisonne sur des faits construits, normalisés, interchangeables qu'elle confronte à des modèles mathématisés ${ }^{27}$. Les arts (de l'artiste, de l'artisan, de l'ingénieur) ont commerce avec des faits actuels. Techniques et arts se rejoignent sur l'impératif de faire et la nécessité de procédures effectives. Plasticiens et ingénieurs ont utilisé, aux mêmes époques, la référence à la science pour rejoindre les arts libéraux.

Le terme "projection ", apparu au XIV ${ }^{e}$ siècle au sens de jeter, est utilisé en géométrie dès le XVII ${ }^{e}$ siècle. Au XIX ${ }^{e}$ siècle, il est utilisé pour décrire le dessin en perspective, les dispositifs d'Alberti et Durer, la camera oscura et la lanterne magique. J.-L. Godard ${ }^{28}$ se livre à une reconstruction qui dit peu de Poncelet, rien de la projection mathématique, mais beaucoup du cinéma (sa relation à la caverne platonicienne, le spectateur prisonnier de son siège, etc.).

Dans une prison de Moscou, J-V Poncelet, officier de l'armée de Napoléon, reconstruit sans l'aide d'aucune note les connaissances géométriques qu'il avait 
apprises dans les cours de Monge et de Carnot. Le Traité des propriétés projectives des figures, publié en 1822, érige en méthode générale le principe de projection utilisé par Desargues pour étendre les propriétés du cercle aux coniques et mis en œuvre par Pascal dans sa démonstration sur l'hexagone mystique.

Il a donc fallu un prisonnier qui tourne en rond en face d'un mur pour que l'application mécanique de l'idée et de l'envie de projeter des figures sur un écran prenne pratiquement son envol avec l'invention de la projection cinématographique. Notons également que le mur de départ était rectangulaire. inévitable vers l'espace subjectif systématique de la Renaissance. Il relie le développement de la perspective avec l'idée kantienne d'un espace infini préexistant à l'objet. Or, une telle définition de l'espace est en cause aujourd'hui. Shepard, soulignant la prégnance de l'interprétation perspectiviste, affirme :

[...] qu'il est impossible de choisir de voir un dessin comme il est, c'est à dire comme une figure composée de lignes sur une surface plane. Si cette figure est conforme aux règles de la perspective linéaire, p. e., elle déclenche automatiquement des circuits cérébraux qui rendent l'interprétation tridimensionnelle appropriée à cette représentation en perspective et nos éventuelles intentions conscientes de laisser une telle interprétation de côté sont impuissantes face aux fulgurantes interventions de ce mécanisme sous-jacent. ${ }^{29}$

Il a la prudence de ne pas se prononcer sur le caractère inné ou acquis des mécanismes qu'il invoque. Depuis plusieurs centaines d'années, l'éducation et de la pratique du dessin perspectif ont obligé les élites occidentales à voir perspectivement. E. Valette ${ }^{30}$, après beaucoup d'autres, s'oppose à la naturalisation de la perspective et à sa réduction à la géométrie projective La géométrie euclidienne prétend décrire l'espace physique qui nous entoure. Elle est une science des figures, ou, en des termes plus modernes, l'étude des invariants par le groupe des transformations «rigides" (rotation, translation, réflexion). Il est donc naturel que l'optique géométrique, en naturalisant le rayon rectiligne, pense les mêmes objets qu'elle: le point, la ligne et le plan. Cette géométrie et cette optique rejoignent les préoccupations d'une peinture qui s'intéresse aux objets et aux figures. Elles n'ont que peu à voir avec les géométries ou la physique modernes.

L'exigence "pointilliste”, calculatrice de Descartes avait été établie dans les Regulae. Elle est au cœur de sa compréhension de la géométrie comme calcul de points (calcul: petite pierre), d'où la numérisation de l'espace cartésien préfigurant l'espace des points du calcul informatique. Or cette réduction de l'espace à un calcul de la position des points dans l'espace est au cœur de l'analyse de l'espace proposée par la perspective et en particulier par Alberti dans son Della Pictura. Avec son velum en grand appareil. [...] L'infrastructure appareillante du cartésianisme, c'est l'appareil de la perspective, la découpe de la pyramide visuelle selon Alberti. Dès lors le modèle de l'identité de la pensée et de l'existence par quoi se définit le cogito se trouve chez Brunelleschi, dans sa démonstration de la perspective... ${ }^{31}$

La compréhension de la géométrie comme calcul des points s'applique bien à la dynamique du point matériel. Du point de vue technique, elle s'applique mal à la statique des structures ou à la perspective, le calcul des points devient rapidement inextricable. Les difficultés de la métrique en perspective ont conduit ingénieurs et architectes à travailler avec d'autres méthodes (géométrie descriptive de Monge, axonométrie) pour résoudre leurs problèmes de tracés.

Si l'appareil perspectif se trouve aujourd'hui intégré aux stations graphiques, c'est par l'intermédiaire de méthodes de projection des modèles dans lesquelles la consistance 
des topologies (voisinage, ordre, etc.) joue un rôle croissant. Alors que la dispute entre Descartes et Desargues a pu paraitre définitivement réglée au bénéfice du premier, la synthèse géométrique mécanisée réhabilite la conception qu'avait Desargues de la géométrie face à la conception cartésienne. Les projections se font le plus souvent en appliquant des transformations géométriques en coordonnées homogènes, donc dans un espace à quatre dimensions. Le $\mathrm{Xx}^{\mathrm{e}}$ siècle $\mathrm{a}$ vu dans un premier temps la remise en cause radicale du programme de Vasari puis l'apparition, grâce à l'ordinateur, du moyen de passer directement d'un langage à la forme sans qu'intervienne un quelconque savoir-faire manuel. L'importance actuelle de la perspective prend sa source dans son caractère algorithmique. La dualité image/algorithme demande à être radicalement saisie : l'imitation disparait pour laisser place à la simulation. Le retour des espaces projectifs en art n'est pas une renaissance de l'appareil perspectif mais l'achèvement de sa liquidation par la banalisation qu'entraîne son incorporation dans des programmes.

\section{L'avant-garde et l'ingénierie}

51 L'avant-garde remet en cause la perspective linéaire. Selon J.-P. Bouillon ${ }^{32}$, les sources de l'inspiration de Kandinsky ne se situaient pas selon la suite reconstruite ultérieurement: \{Schönberg / relativité et théorie des quanta / abstractions\}, mais étaient \{Wagner / la perte de l'intégrité de l'atome ${ }^{33}$ / la peinture pure de Monet\}. Dans une critique proche des idées révolutionnaires, Lissitzky et Malevitch identifiaient la perspective linéaire à l'individualisme bourgeois. Contre la perspective, Malevitch défendait la libre circulation des formes et Lissitzky l'axonométrie. Ils se libéraient ainsi du point de vue unique, du point de vue de la singularité. Avec l'axonométrie, Lissitzky se rapprochait de l'ingénierie, mais surtout déplaçait l'observateur, devenu multitude, à l'infini. Florensky, défenseur réactionnaire et clérical de la spécificité russe et de l'icône critiquait lui aussi l'individualisation de la vision par la perspective. Le paradoxe n'est qu'apparent. Dieu aussi regarde le monde de l'infini.

La relation de l'avant-garde à la théorie de la relativité et à la physique quantique est largement anachronique. Un examen sérieux de certaines déclarations, révèle que c'est souvent la relativité galiléenne qui est décrite. Ce n'est ni du côté des physiciens, ni de celui des mathématiciens qu'il faut chercher des sources directes. Dès le dernier quart $\mathrm{du} \mathrm{xIX}^{\mathrm{e}}$ siècle, les géométries, non-euclidiennes à quatre dimensions et plus, commencent à se diffuser. Ce ne sont pas Klein, Riemann ou Hilbert qui alimentent les réflexions des artistes, mais Hinton, Abbott, Ouspensky et Jouffret ${ }^{34}$. Les artistes du début $\mathrm{du} \mathrm{xx}^{\mathrm{e}}$ siècle ont accès aux géométries non euclidiennes, dans des versions vulgarisées, en particulier à partir des œuvres de Charles Howard Hinton ${ }^{35}$ ou du livre d'Edwin A. Abbott ${ }^{36}$ décrivant le comportement d'individus dans un pays plat. En Russie, ces théories pénètrent par l'intermédiaire de P.-D. Ouspensky, mathématicien et auteur connu puis disciple de Gurdjieff. La suite de cette histoire ne se retrouvera pas dans les manuels de science mais bien dans l'ésotérisme, chez les anthroposophes et dans la littérature de science-fiction. En France, la diffusion des géométries non euclidiennes, parmi les peintres cubistes, se fait au travers du livre de Jouffret ${ }^{37}$, dont l'exposé, beaucoup plus dans la tradition de la géométrie, portera moins à des extrapolations ésotériques. Très peu d'artistes ont eu une relation à un scientifique aussi forte que celle qui lie Duchamp à Poincaré. Duchamp aborde des thèmes comme le hasard, les espaces à quatre dimensions, la linguistique etc. Le « Grand Verre » est une 
tentative de synthèse de ces théories. Lyotard a décrit comment Duchamp utilise le principe géométrique pour cette œuvre majeure. Il lui a emprunté une description, inspirée de Poincaré de la construction de l'espace à quatre dimensions ${ }^{38}$. Le Duchamp de la série Les Joueurs d'échecs connaissait le livre de Jouffret auquel il emprunte le concept d'« épanouissement » comme extension dans une autre dimension. Il a aussi reconnu s'être inspiré d'un livre d'anticipation de Gaston de Pawlowski ${ }^{39}$.

À l'opposé de la tradition grecque ou judéo-chrétienne, la fin du XIX ${ }^{\mathrm{e}}$ siècle commence à reconnaître l'image comme instrument de la raison. En 1877, le statisticien Francis Galton utilise des portraits composites pour montrer des catégories abstraites permettant de visualiser des types humains universels (la prostituée, le criminel, l'Anglais, le Juif, etc.). En 1880, les lignes fermées de John Venn (les "patatoïdes » utilisés dans l'enseignement de la théorie des ensembles) permettent de traiter certaines catégories logico-mathématiques. En 1900 Sigmund Freud, dans L'Interprétation des rêves, propose une nouvelle compréhension de la vision qui n'est pas inférieure à la raison mais au contraire, son égale comme moyen de représenter des idées abstraites ou des arguments logiques. La figure devient son propre objet. Elle représente des idées, des concepts abstraits et pas des projections, elle se détache de ce que notre œil peut ou pourrait voir. (Malevitch affirmant l'autonomie de la peinture, Duchamp critiquant la peinture rétinienne). Le cinéma d'Eisenstein renforce la connexion historique entre l'intérêt pour le raisonnement visuel et l'émergence de la communication de masse.

C'est dans la relation à une technique, toujours plus pénétrée par les méthodes et concepts issus de la science, qu'il faut rechercher les clés. L'avant-garde, a été le fer de lance d'un modernisme d'ingénieur. Elle reprend à son compte les paradigmes de l'ingénierie moderne: précision, sûreté, fiabilité, vitesse, simplicité, efficacité, économie. Les futuristes comme Marinetti vantent le machinisme et la vitesse. Lazlo Moholy-Nagy a plaidé la nécessité de concevoir précisément (engineer) la photographie publicitaire :

Un ingénieur moderne, si les objectifs et les aspects fonctionnels de son travail sont clairs, peut, sans grand effort faire un produit formellement adéquat et parfait dans sa construction économique. Mais la publicité photographique actuelle n'est pas si facile à définir. Il n'existe pas de "manuel de l'utilisateur». La recherche dans les lois physiologiques et psychologiques de l'efficacité visuelle demeure loin derrière, comparée à l'étude des lois physiques. ${ }^{40}$

Gabo compare son travail à celui de l'ingénieur ou du mathématicien. « Le fil à plomb dans nos mains [...] nous construisons notre œuvre [...] comme l'ingénieur construit les ponts, comme le mathématicien élabore les formules des orbites $\star^{41}$. Les modes de pensée du concepteur industriel et de l'artiste répondant à une commande sont proches. L'impératif est de faire et non de connaître et ils n'innovent qu'en transgressant. Analogique, la pensée du créateur est fondée sur la perception. Une solution acceptable doit être trouvée dans un délai donné et avec des moyens limités. L'art du technicien est de déterminer le moment où les conditions d'une décision sont mûres. L'instrument de base de cet art est le jugement. L'entendement analyse, dissèque et détaille, le formalisme logique n'intervient qu'une fois la solution trouvée, pour la confirmer ou la réfuter. 


\section{Le postmodernisme et la complexité}

56 La physique échappe de plus en plus aux laïcs, mais pénètre le public sous la forme d'un scientisme vulgarisé que l'on peut qualifier d'hyperscience. C'est le produit naturel de la science et les scientifiques sont toujours complices de sa diffusion. Cette complicité est à la mesure des budgets demandés. La vision "populaire » de la science devient aussi importante que la science elle-même. Que serait la physique contemporaine sans la personnalité d'Einstein, sans la simplicité de la formule $\mathrm{E}=\mathrm{MC}^{2}$ concrétisée par la bombe atomique, sans l'affirmation banalisée que «tout est relatif », sans l'application à tort et à travers du principe d'incertitude de la théorie quantique.

La "théorie du tout ", que nous promettent les physiciens théoriciens contemporains, est l'ultime tentative de la physique de poursuivre sa domination. Le choix d'une dénomination idéologique pour une unification théorique, qui sans cela resterait totalement abstraite pour l'honnête homme contemporain, est délibéré. Les physiciens des hautes énergies ont des besoins croissants, le produit de la taille de la particule par celle de l'outil de recherche tend à être constant. Ils ont besoin d'exister dans une opinion publique moins effrayée par l'apocalypse nucléaire depuis l'effondrement de l'URSS. Un développement singulier s'achève. Si la domination de la technoscience se poursuit, elle n'est plus celle du complexe militaro-industriel. La physique subit aujourd'hui la concurrence de la biologie. Manipulant le vivant, les biologistes nous promettent un avenir de rêve ou de cauchemar. La microbiologie se veut la «big science » de demain.

La science tend à prendre en compte la complexité, écartée par Galilée. Elle se rapproche de l'évènement rare, singulier. L'irruption de la complexité et ses conséquences jouent un rôle central dans la postmodernité. La pensée moderne était une pensée de la régularité et de la reproduction des phénomènes. La pensée contemporaine s'ouvre aux irrégularités, aux exceptions et prend en compte le caractère bruité de l'univers. Non bruité l'univers devient illisible et inaudible ou alors transcendantal. La vision humaine, ajustée sur la vision de la nature, intègre sans difficulté la complexité si toute structure ne disparaît pas. Notre relation à un authentique, qui ne réside pas dans la pureté platonicienne, change profondément. Authenticité et aura résident probablement dans la part du «bruit» qui fait sens. On raconte que Heisenberg aurait déclaré sur son lit de mort qu'il poserait à Dieu deux questions : pourquoi la relativité et pourquoi la turbulence? Il aurait ajouté être persuadé que Dieu répondrait à la première question. ${ }^{42}$

Toute empreinte ou trace est bruitée (effets aléatoires de flou, d'effacement de détails, de bruits induits par le procédé). Il n'y a pas coupure nette mais zone d'incertitude, de turbulence. Analogique et conduite de façon correcte, la prise d'empreinte, la saisie de la trace, ne crée pas d'artefacts inacceptables. Le découpage de l'espace et du temps, à l'aide de machines finies, ce que l'on nomme échantillonnage, génère par son acuité même, par la rigueur de l'exécution de son programme, des artefacts massifs et structurés (les bruits d'échantillonnage). Au cinéma, la quantification du temps perturbe certains mouvements par un effet stroboscopique (la roue de la diligence qui tourne à l'envers). En vidéo, la dimension fixe du pixel crée des effets de moiré si l'on filme certaines structures en grille. Pour les images de synthèse, il y a génération d'aberrations d'ordre topologique. Le caractère fini des machines et des procédés fait irruption dès que le programme tente de sortir l'idéal du monde des idées. 


\section{La question de l'appareil et du « faire époque »}

\section{Industries culturelles et outils, programmés}

60

hui, les supports (argentique, vidéo, infographique) se mélangent. L'image acquise sur pellicule est ensuite numérisée et/ou diffusée en vidéo, des images infographiques ou vidéo sont transférée sur pellicules. La bigarrure n'est que partielle, il y a domination du langage cinématographique dans sa version appauvrie, hollywoodienne. Les méthodes et procédures codées dans les machines imitent le média dominant, allant jusqu'à reproduire les aberrations optiques des caméras et même le reflet du diaphragme sur la lentille frontale. La mise en œuvre des moyens numériques est utilitariste : réaliser à moindres frais des effets spéciaux ornementaux ou catastrophiques, réaliser des décors, corriger des défauts (comme éliminer l'ombre d'une perche), reconstituer des scènes sans avoir à convoquer les équipes, etc. La modélisation 3D permet d'envisager des mouvements de caméras presque infaisables en animation traditionnelle, mais elle permet surtout d'éliminer le recours à certains savoir-faire. Toutes ces pratiques, s'intégrant à un système de simulation de plus en plus complexe, ne mènent qu'au simulacre.

61 La composition d'images, par des moyens numériques (dits d'editing / compositing), est facilitée si l'on connaît en tout temps la position de la caméra (on manipule ainsi un morceau de plan isomorphe à son plan focal). Le story board devient un programme qu'une machine de motion control exécute, asservissant le mouvement de la caméra. Les cinéastes de la "Nouvelle Vague » avaient libéré la caméra de son pied ou de son chariot. Jonas Mekas en avait fait le prolongement de la main et non une prothèse de l'œil. L'industrie cinématographique fait, de plus en plus souvent, intervenir dès le tournage les équipes de postproduction. L'exécution en parallèle d'activités traditionnellement organisées de façon séquentielle fait de toute remise en cause du programme initial une catastrophe. Elle abolit les tensions, les périodes de remise en cause de l'écriture ou de la lecture que l'on en fait. L'écriture initiale devient programme, rien ne peut plus arriver. Il ne reste plus qu'à constater la mort d'un cinéma réifié à ce point.

La technique industrielle met en œuvre un programme, pour obtenir un produit L'utilisation d'outils, programmés de façon à inclure un modèle des savoir-faire des industries culturelles, confronte l'artiste à la question : faire une CEuvre ou un produit? Si le produit résulte de l'application du programme, l'œuvre démasque le programme. Des pratiques nouvelles sont possibles, mais les industries de l'imaginaire jouent la tyrannie de l'uniformité et du "même partout». La courte histoire des tentatives artistiques utilisant l'ordinateur peut suggérer que c'est en détournant le simulacre, ou en s'écartant de la mise en œuvre des machines géométriques créées pour l'industrie, que se trouve l'avenir d'un art utilisant l'ordinateur.

\section{L'image numérique ne fait pas époque}

L'ordinateur et ses différentes déclinaisons sont les objets phares d'une métamorphose du travail, des loisirs et des industries culturelles. Même s'il n'y a pas d'exemple, dans l'histoire de l'art, d'une évolution technique et scientifique capable de transformer en 
profondeur notre perception du monde, qui n'ait fini par engendrer une nouvelle forme symbolique, l'ordinateur en tant que tel ne fait pas plus époque que l'automobile.

Le peintre fractaliste J.-P. Agosti a affirmé « Les nouveaux médias comme le laser ou de nouvelles théories comme les fractales de Mandelbrot sont pour moi des outils que j'utilise dans l'élaboration d'une esthétique de mon époque ». Il oublie que dans la relation entre l'outil et la pensée ce n'est pas inévitablement la pensée qui dirige l'outil. Il arrive que l'outil conditionne la pensée et c'est particulièrement vrai pour les outils informatiques qui incorporent la pensée de ceux qui les programment. Le chercheur en informatique J.-P. Allouche souligne : « Le délai avec lequel les objets mathématiques se glissent dans les boîtes à outils des artistes (Pi, Fibonacci, automates finis, automates cellulaires, objets fractals, théorie des cordes, modules de Drinfeld, analyse nonstandard) est de plus en plus court $\aleph^{43}$. La vitesse d'usure des images mathématiques, en partie due au nombre de déclinaisons du traitement de tout objet intéressant, est impressionnante. On se lasse vite des effets spectaculaires au premier abord qu'elles produisent: surfaces réglées des modernes, surfaces minimales et bulle de savon, surfaces topologiquement étranges (ruban de Möbius et bouteille de Klein). Man Ray, dans les années trente a produit de très belles photos de surfaces géométriques matérialisées par des maquettes. Ces photographies doivent leur qualité à la lumière de Man Ray, les objets qui sont leurs référents ne sont plus regardables aujourd'hui.

L'écran du téléviseur (l'« étrange lucarne » selon la vieille formule du Canard Enchaîné) ou celui de l'ordinateur peuvent être considérés comme une fenêtre ouverte sur l'appareil mondial de diffusion de l'information et des productions de l'industrie culturelle. Les systèmes se développent en fonction des besoins de cette industrie. Ils ne cherchent pas des formes nouvelles, mais visent à occuper le terrain en étendant un peu les possibilités techniques des dispositifs, mais surtout en produisant et en distribuant, à moindres coûts, des images toujours plus spectaculaires. Le retour massif du direct à la télévision est le phénomène le plus pertinent, justifiant une analyse fine. Les capacités de manipulation en "temps réel » ne signifient pas l'accès en direct étendu à la planète. Elles sont, au contraire, l'abolition du direct par l'interposition, qui peut rester cachée, d'un système technique complexe. La manipulation de systèmes programmés se substitue au montage au travers de l'interactivité ou dans les nouvelles émissions de plateau. Ce retour aux sources de la télévision, cette réduction du montage à "l'habillage» et à l'élimination de scories est la nouvelle esthétique télévisuelle. L'immédiateté est exigée : la TV formule des questions et y répond en quelques dizaines de secondes, au-delà c'est l'ennui. Les temps de la vie, de la réflexion et du partage de la réflexion avec l'autre sont abolis. Le spectacle est permanent et immédiat, il s'oublie d'une séance à l'autre. Le temps se trouve réduit à l'immédiat, le passé est oublié, le futur indifférent.

\section{Autour de programmes et réseaux : l'émergence d'un nouvel appareil}

Les signes de l'émergence d'un appareil nouveau, s'articulant sur programmes et réseaux, sont nombreux. Savoir s'ils sont décisifs fait débat. Le caractère industriel des techniques renforce leur intégration à l'ordre social, donc leur dépendance à cet ordre. Les virtualités des techniques ne sont pas nécessairement actualisées. Les germes des dispositifs techniques massivement utilisés aujourd'hui existaient en 1950. Pourtant en 1950, l'an 2000 était anticipé comme l'ère de la locomotion individuelle généralisée. 
L'industrie cherche à retrouver dans l'exécution des programmes la souplesse et la plasticité qu'avait leur mise en œuvre.

Partant des divergences entre temporalités politiques et temporalités médiatiques, Vitalis ${ }^{44}$ conclut qu'il n'y a pas de formes politiques innovantes à attendre des nouvelles technologies. Pour lui, les espaces publics ainsi créés sont : « déconnectés des processus de décision et donc sans débouché proprement politique ». La télévision, média dominant sur le plan politique et social, n'est pensable aujourd'hui que dans l'articulation de programmes et de réseaux précisément fondés sur les nouvelles technologies. Même limité à l'Internet, l'argument de Vitalis n'est pas recevable. En effet, les formes prises par les mobilisations contre la mondialisation ne peuvent être comprises sans l'intervention des réseaux. L'affirmation de Benjamin sur la technique émancipée est plus vraie que jamais :

Cette technique émancipée se trouve maintenant en face de la société actuelle comme une seconde nature et notamment, ainsi prouvent les guerres et les crises économiques, comme une nature non moins élémentaire que celle qui était donnée à la société des origines. L'homme se trouve devant cette deuxième nature qu'il a bien inventée, mais qu'il ne maîtrise plus, déjà depuis longtemps, suivant son enseignement, comme jadis devant la première nature. Et à nouveau, l'art se trouve au service de cette nature. ${ }^{45}$

68 Les actions humaines d'appropriation et de transformation de la nature ont affecté l'écosystème dans son ensemble à tel point que la différence entre nature et artifice semble se dissoudre et l'ensemble du réel nous apparait composé de choses qui sont naturelles et artificielles à la fois. Le rapport nature/artifice se modifie profondément lorsque la simulation de la nature remplace son imitation. Les réseaux qui innervent la société, les systèmes informationnels actuels, remettent en cause les rapports que nous entretenons avec l'espace et le temps. Face à la nature l'homme se mouvait à la surface d'un espace perçu tridimensionnel. Le cosmos était éternel infini et indéfiniment sécable. Les physiciens de la théorie des cordes et supercordes considèrent un cosmos fini et réticulé. Le développement des technologies entraîne un élargissement et une dissolution du concept d'espace: espaces d'états, espace visuel, espace de communication (en particulier celui des réseaux), espace culturel. Les réseaux tissent un filet (net) qui laisse de moins en moins de possibilités d'emprunter des chemins de traverse. Au continuum tridimensionnel euclidien succède un ensemble d'espaces unidimensionnels et réticulés: les réseaux de transports (routes, chemins de fer, couloirs aériens) sont les espaces quasi exclusifs de nos déplacements, les réseaux télématiques documentent nos activités, mais aussi nos inclinations et une partie de nos désirs.

69 Le multimédia est la réduction à une surface d'inscription unique : l'espace scalaire des textes et des codes. Avec l'image numérique, il ne s'agit plus de replier le temps sur l'espace (cinéma) ou de coder l'espace sur le temps (vidéo) : l'image devenue texte ${ }^{46}$ peut être manipulée sans limites. On combine des images acquises avec des images créées sans qu'il soit possible de les distinguer les unes des autres. L'image numérique présente un caractère mimétique, mais de trace ou empreinte, elle devient compte rendu, son lien au réel, à un référent, facultatif. L'œuvre numérisée n'est plus directement accessible, elle n'est que virtuelle. Avant la généralisation des dispositifs d'enregistrement, l'œuvre musicale inscrite sur sa partition ne devenait accessible au laïc, incapable de l'apprécier par la lecture, qu'au travers de l'interprétation. L'œuvre numérisée n'est actualisée que par l'exécution, par une machine, de la procédure 
décrite par un code. Pire, l'accès au code lui-même a perdu toute pertinence. Décomposé jusqu'à un niveau où il ne porte plus aucun ancrage signifiant dans le réel, il n'est que conventions. Le texte codant les procédures d'actualisation d'un objet virtuel devient la surface d'inscription, totalement banalisée, de l'art comme de la plupart des activités humaines. Cette nouvelle surface d'inscription est scalaire et éclatée car le code peut être dispersé dans un ensemble de systèmes informatiques. Les interdépendances croissantes tendent à étendre la surface d'inscription au réseau mondial des ordinateurs. En fait d'accès à la chose, le spectateur accède à une instanciation de l'œuvre, échappant à l'artiste car soumise à l'évolution industrielle.

\section{En conclusion provisoire}

Comment envisager la naissance d'une esthétique nouvelle? En tout cas pas comme Mario Costa qui rejoint la longue liste de ceux qui ont proclamé la fin de l'histoire de l'art:

Je crois que l'histoire de l'art est historiquement conclue, mais je crois aussi que l'expérience esthétique ne peut pas encore être éliminée de la configuration actuelle de l'humain et qu'il faut la rechercher dans la mise en œuvre, au moyen des technologies, d'une nouvelle sorte de sublime.

71 Le "sublime technologique " de Costa est créé par l'attraction/répulsion, peur/ domination que suscitent les nouvelles technologies. Domestiqué, ce sublime n'est plus un sentiment individuel issu d'une situation fortuite mais un sentiment issu d'une situation collective planifiée. Il peut être ressenti au travers de l'art. À la différence du sublime de Lyotard, le sublime technologique de Costa est le triomphe de la raison et de la domination de notre intellect logico-mathématique. J'affirme au contraire que ce "logico-mathématique ", que je persiste à nommer "programme », banalise tout ce qu'il incorpore.

Les méthodes de la technique s'articulaient sur deux pratiques fondamentales :

- déformer ou abraser un milieu continu (continuum, rôle de la surface d'inscription) : ce qui renvoie aux infinis cantoriens, à la plasticité \{modelage, trace, empreinte\},

- couper-coller des objets ou des séquences de signes (systèmes discrets, écriture, rôle contingent de la surface d'inscription) : ce qui renvoie aux combinatoires, aux codes et aux langages \{écritures, montage, mixage\}.

73 L'analyse et l'action autour de l'espace et du temps ne sont plus fondées sur le continuum. Quantification finie et codage dissolvent la différence des deux pratiques dans la manipulation "mécanique » de langages relativement formalisés. L'appareil émergent est caractérisé par :

- la domination de programmes fondés sur des modèles formalisés à la recherche d'une " généricité » croissante,

- le remplacement du modèle cybernétique et sa contre-réaction par l'anticipation et les automatismes « a priori ",

- la réduction de l'espace aux mondes unidimensionnels des réseaux maillés, du temps à l'immédiateté des actions dites en « temps réel ",

- l'inscription de toute action humaine dans l'espace scalaire de codes dont toute actualisation exige un dispositif technique complexe. d'échapper à un utilitarisme encore plus radical que celui du $18^{\mathrm{e}}$ siècle anglais ou au 
détour par le sublime, technologique ou non. Le triplet \{dispositif technique complexe / modèle théoriquement fondé / ensemble de données informationnelles\} permet de désigner un appareil de simulation. La polysémie du terme simulation rend difficile son adoption pure et simple. La simulation renvoie à des ressorts psychologiques profonds, au « faire-semblant » des enfants ou à la production / consommation de fictions chez les adultes. L'étude des capacités humaines de mentalisation (c'est-à-dire d'attribution à soi et à autrui d'états mentaux), montre que le développement progressif de la capacité d'anticipation évoquée au début de ce texte, peut transformer des représentations de croyances et de désirs d'autrui, non pas en décisions d'agir, mais en prédictions sur les décisions d'agir d'autrui. La simulation est en rapport étroit à la représentation, donc à la question de la mimèsis, que les artistes modernes ont contribuée à déconstruire en se donnant pour objet leur propre langage. La vision platonicienne établissait une hiérarchie entre les imitations de la nature par les arts : au plus bas peinture et musique élaborant des simulacres, au plus haut, les arts " sérieux ", recourant au nombre et à la mesure lieu de rencontre entre l'imitation et l'ordre mathématique. Ces derniers participaient à la métaphore du démiurge. Si certains prennent encore au sérieux une telle hiérarchie, il faut constater aujourd'hui que mathématiques et mesures ne font en rien échapper au simulacre. Les scientifiques et les ingénieurs recourent de plus en plus à la simulation devenue outil de découverte utilisé pour élaborer des résultats scientifiques. Elle devient forme d'expérimentation et source d'une nouvelle représentation du réel. L'interprétation sensible d'images devient la seule façon de faire face aux avalanches de résultats. L'image permet de visualiser le concret comme l'abstrait, le directement perceptible comme l'imperceptible (le mouvement d'un fluide coloré aussi bien que les échanges sur l'Internet), mais inscrite dans un dispositif d'imagerie, elle perd tout intérêt esthétique. C'est au carrefour des différents sens de ce terme et dans la mesure où tous ses aspects sont devenus objets de techniques convergentes, que l'on peut envisager la simulation généralisée comme un appareil.

\section{BIBLIOGRAPHIE}

Abbott E. A., Flatland, Paris, Denoël, 1998.

Allouche J.-P., L'art technologique est-il nouveau? Alliage, n³3-34, 1998.

Bachelard G., Essai sur la connaissance approchée, Paris, Librairie J. Vrin, 1981.

Barthes R., Sade, Fourier, Loyola, Paris, Seuil, 1971.

Benjamin W., L'œuvre d'art à l'époque de sa reproductibilité technique, Allia, Paris, 2003.

Berthoz A., Le sens du mouvement, Paris, Odile Jacob, 1997.

Biezunski M., Histoire de la physique moderne. Paris, La Découverte, 1993.

de Pawlowski G., Voyage au pays de la quatrième dimension, Paris, Denoël, 1923.

Déotte J.-L., L'époque des appareils, Paris, Éditions Lignes \& Manifestes, 2004. 
Feyerabend P., Dialogues sur la connaissance, Paris, Seuil, 1996.

Foucault M., Raymond Roussel, Paris, Folio Gallimard, 1992.

Freud S., Malaise dans la civilisation, Paris, PUF, Bibliothèque de psychanalyse, 1973.

Gabo N., Manifeste réaliste, 1920.

Godard J.-L., Histoire(s) du cinéma, Paris, Gallimard, 1998.

Goodman N., L'art en théorie et en action, Paris, édition de l'Éclat, 1996.

HabermasJ., La technique et la science comme « idéologie », Paris, Gallimard, 1973.

Henderson L. D., The Fourth Dimension and non Euclidian Geometry In Modern Art, Princeton, Princeton University Press, 1983 (en voie de réédition).

Jouffret E., Traité élémentaire de géométrie à quatre dimensions et introduction à la géométrie à $n$ dimensions, Paris, édition Gauthier-Villars, 1903.

Kandinsky V., Regards sur le passé et autres textes 1912-1922, Paris, Hermann éditeurs des sciences et des arts, coll. « Savoir », 1974.

Kant E., Critique de la Raison pure, Paris, Flammarion, 1976.

Klein F., Le Programme D'Erlangen. Considérations comparatives sur les recherches géométriques modernes, Paris, Gauthier-Villars, 1974.

Kloepfer R., «La puissance des prémisses anthropologiques dans la théorie du film de Sergueï M. Eisenstein ", in Eisenstein. L'ancien et le nouveau, D. Château (dir.), Paris, Publications Sorbonne/ Colloque de Cerisy, 2001.

Lyotard J.-F., Les Transformateurs Duchamp, Paris, Galilée, 1977.

Moholy-Nagy L., "Photography in Advertising", in Photography in the Modern Era, New York, ed. Christopher Phillips Aperture, 1989.

Malevitch K., Écrits, présentés par A. Nakov, Paris, éditions Ivrea, 1996.

Païni D., Projections, les transports de l'image, Hazan / Le Fresnoy / AFA, 1997.

Porchet M., La production industrielle de l'image, Paris, l'Harmattan, coll. « Esthétiques », 2002.

Risset J.-C., «Perception, environnement, musiques », InHarmonique, $\mathrm{n}^{\circ}$ 3, « Musique et perception », Paris, Ircam, mars 1988.

Segalen V., Équipée : voyage au pays du réel, Paris, Gallimard, 1983.

Shepard R. N., L'œil qui pense, Paris, Seuil, 1992.

Simon H., The Sciences of the Artificial Intelligence, Cambridge, MIT Press, 1969.

Valette E., La perspective à l'ordre du jour, Paris, l'Harmattan, coll. « Esthétiques », 2001.

Vitalis A., Tétu J.-F., Palmer M., Castagna B. (dir.), Médias, temporalités et démocratie, Rennes, éditions Apogée/PUF, 2000.

Wittgenstein L., Remarques mêlées, Paris, éditions TER, 1990. 


\section{NOTES}

1. Le professionnel compétent, efficace, fidèle à la mission qu'on lui confie et sans état d'âme est au cœur de l'idéologie américaine. "On est là pour faire un boulot et c'est tout " (déclaration d'un militaire américain en Irak en mars 2003).

2. J. Habermas, La technique et la science comme « idéologie », Paris, Gallimard, 1973.

3. Limites décrites dans S. Freud, Malaise dans la civilisation, Paris, PUF, Bibliothèque de psychanalyse, 1973.

4. G. Bachelard, Essai sur la connaissance approchée, Paris, Librairie J. Vrin, 1981.

5. Je laisse de côté Jacques Ellul, qui a vu l'importance de la jonction entre techniques du spectacle et techniques de production dans les nouvelles technologies. Mais il considère la technique, en fait réduite aux techniques industrielles, comme un système dominant global et autonome. Il soumet ainsi la technique à un finalisme auquel son développement réel échappe.

6. Cette méthode consiste à ajuster à une situation les paramètres d'une "idéalité " posée a priori.

7. R. Barthes, Sade, Fourier, Loyola, Paris, Seuil, 1971.

8. C'est une caractéristique des problèmes que les mathématiciens nomment NPdurs ou NPcomplets (NP pour Non Polynomiaux) dont la décomposition débouche sur au moins un problème aussi complexe que le problème initial.

9. H. Simon, The Sciences of the Artificial Intelligence, Cambridge, MIT Press, 1969.

10. L. Wittgenstein, Remarques mêlées, Paris, éditions TER, 1990.

11. V. Segalen, Équipée : voyage au pays du réel, Paris, Gallimard, 1983.

12. J.-C. Risset, "Perception, environnement, musiques ", InHarmonique, $\mathrm{n}^{\circ} 3$, « Musique et perception », Paris, Ircam, mars 1988.

13. Voir à ce propos, P. Feyerabend, Dialogues sur la connaissance, Paris, Seuil, 1996.

14. F. Klein, Le Programme D’Erlangen. Considérations comparatives sur les recherches géométriques modernes, Paris, Gauthier-Villars, 1974.

15. E. Kant, Critique de la Raison pure, Paris, Flammarion, 1976.

16. A. Berthoz, Le sens du mouvement, Paris, Odile Jacob, 1997.

17. W. Benjamin, L'œuvre d'art à l'époque de sa reproductibilité technique, Allia, Paris, 2003.

18. Idem.

19. Interview de Chris Marker dans Libération Cinéma du 5 mars 2003.

20. Idem.

21. Eisenstein cité par R. Kloepfer, « La puissance des prémisses anthropologiques dans la théorie du film de Sergueï M. Eisenstein ", in Eisenstein. L'ancien et le nouveau, D. Château (dir.), Paris, Publications Sorbonne/Colloque de Cerisy, 2001.

22. K. Malevitch, Écrits, présentés par A. Nakov, Paris, éditions Ivrea, 1996.

23. Qui a trouvé une réalisation particulièrement radicale dans l'EVE de Jeffrey Shaw.

24. D. Païni, Projections, les transports de l'image, Hazan / Le Fresnoy / AFA, 1997.

25. M. Foucault, Raymond Roussel, Paris, Folio Gallimard, 1992.

26. N. Goodman, L'art en théorie et en action, Paris, édition de l'Éclat, 1996.

27. Parlant de l'actuel, le savant se fait expert, il devient sa propre caricature. De la spéculation régulée par le débat avec ses pairs, il passe à l'affirmation dans les médias ou devant les tribunaux. Hors de quelques théoriciens, les scientifiques jouent le jeu, et pas seulement pour flatter un ego aussi surdimensionné que celui des artistes.

28. J.-L. Godard, Histoire(s) du cinéma, Paris, Gallimard, 1998.

29. R. N. Shepard, L'œil qui pense, Paris, Seuil, 1992.

30. E. Valette, La perspective à l'ordre du jour, Paris, l'Harmattan, coll. « Esthétiques », 2001.

31. J.-L. Déotte, L'époque des appareils, Paris, Éditions Lignes \& Manifestes, 2004. 
32. V. Kandinsky, Regards sur le passé et autres textes 1912-1922, Paris, Hermann éditeurs des sciences et des arts, coll. « Savoir ", 1974.

33. La découverte de l'électron sépare l'atome des physiciens de l'atome indivisible de Leucippe, Démocrite et leurs successeurs.

34. Voir la monumentale étude de L. D. Henderson, The Fourth Dimension and non Euclidian Geometry In Modern Art, Princeton, Princeton University Press, 1983 (en voie de réédition).

35. La contribution d'Hinton aux mathématiques est restée très modeste, par contre, le terme «Tesseract » qu'il utilise pour décrire un hypercube non développé aura le plus grand succès dans la littérature de science-fiction et dans l'ésotérisme.

36. E. A. Abbott, Flatland, Paris, Denoël, 1998.

37. E. Jouffret, Traité élémentaire de géométrie à quatre dimensions et introduction à la géométrie à $n$ dimensions, Paris, édition Gauthier-Villars, 1903.

38. J.-F. Lyotard, Les Transformateurs Duchamp, Paris, Galilée, 1977.

39. G. de Pawlowski G., Voyage au pays de la quatrième dimension, Paris, Denoël, 1923.

40. L. Moholy-Nagy, "Photography in Advertising", in Photography in the Modern Era, New York, ed. Christopher Phillips Aperture, 1989.

41. N. Gabo, Manifeste réaliste, 1920.

42. Cette anecdote, rapportée par James Gleick, est citée par M. Biezunski, Histoire de la physique moderne. Paris, La Découverte, 1993.

43. J.-P. Allouche, L'art technologique est-il nouveau ? Alliage, $\mathrm{n}^{\circ} 33-34,1998$.

44. A. Vitalis, J.-F. Tétu, M. Palmer, B. Castagna (dir.), Médias, temporalités et démocratie, Rennes, éditions Apogée/PUF, 2000.

45. W. Benjamin, ouvrage cité ci-dessus.

46. M. Porchet, La production industrielle de l'image, Paris, l'Harmattan, coll. « Esthétiques », 2002.

\section{RÉSUMÉS}

Partant de l'idée que la technique n'est affaire ni d'infinité ni de vérité, cet essai interroge les conséquences de la technique et de la technoscience sur l'expérience du corps, de l'espace et du temps. En montrant le procédé (le rush, la bande vidéo, le programme), il interroge les manipulations du temps et de l'espace propres au film, celles de la vidéo, puis l'immersion des univers virtuels, qu'il distingue des installations. Refusant de suivre les chronologies de l'histoire de l'art qui minimisent la perspective dans la création artistique moderne, il met en avant l'importance actuelle, algorithmique, de la perspective qui ne sert plus l'imitation mais la simulation.

\section{INDEX}

Mots-clés : cinéma, vidéo, virtuel, montage, ordinateur, corps, simulacre 